\title{
EDITORIAL
}

\section{A Shift in Focus}

\author{
Ra-id Abdulla ${ }^{1}$
}

Received: 25 February 2018 / Accepted: 2 March 2018 / Published online: 10 April 2018

o) Springer Science+Business Media, LLC, part of Springer Nature 2018

The Heart Innovation Forum, an AHA-sponsored conference hosted an unexpected speaker in its Fall 2017 meeting. The speaker, Jackson (Jack) Radant, was eloquent, effective, inspiring, and very young. He must have been no more than 18 years old, yet he provided unrivaled wisdom and experience. Jack talked about living with hypoplastic left heart (HLH) syndrome, not as a physician, but as a patient who survived three stages of Fontan procedure, heart failure, LVAD, and finally cardiac transplantation. Jack had experience we as health care providers lack. His positive disposition enabled him to envision how things could be better and his fluency of expression allowed his message to be clear and effective. Therefore, I was pleased when he agreed to my offer to narrate his experience though an editorial for Pediatric Cardiology.

Jack's talk and editorial caused me to reflect on the increasing percentage of older children and adults in pediatric cardiology practices, a testament to improved medical and surgical care of children with congenital heart diseases. I know most of my patients and their families since birth, allowing me to establish a strong bond and a rewarding experience as I attend to their medical needs throughout their teenage years and even into adulthood. Many of these patients are now in their 20s and 30s; some of them have become parents themselves and most are healthy and able to accomplish all they aspire to do. Unfortunately, a small but growing sector of this population has not fared as well. Patients with single ventricle, particularly HLH and its variants continue to pose a challenge in management and become a source of frustration to patients and health professionals. As these patients enter into their teenage years, it is expected that many of them will develop complications secondary to the Fontan procedure. Arrhythmias and heart failure as these teens get older become a more likely scenario in

Ra-id Abdulla

Ra-id_Abdulla@rush.edu

1 Rush University, 1630 W Harrison Street, Chicago, IL 60607, USA the majority of these patients and eventual referral for heart transplantation is becoming inescapable for many.

Earlier in my career I believed, or at least hoped, that the single ventricle palliation for HLH would have delivered a better outcome than the one I currently observe. Diagnosis at or even before birth, prompt intervention, and the amazing advancement in surgical, post-operative, and pharmacological improvements did indeed improve outcome and survival. However, a single right ventricle is unlikely to allow normal life style in most patients into middle age. Therefore, all that is done in childhood years for these patients is to postpone the inevitability of heart failure to a later age when no medical or surgical intervention can rescue the failing right ventricle and heart transplantation becomes the only option available.

The Fontan procedure is a definitive step in an attempt to palliate single ventricle physiology. Complete surgical repair of most other congenital heart disease entails a finality of the repair process where redo surgeries to upgrade valves or deal with complications may be an occasional need; this is not the case in single right ventricle patients with a Fontan procedure. Heart failure and the need for cardiac transplantation is a more likely scenario than sailing into middle age with just the Fontan procedure. Therefore, I am growing increasingly uncomfortable with the prevailing notion that the Fontan procedure, particularly in HLH patients is in any way the final step of a three-stage plan. A more realistic approach is to consider the Fontan procedure as the third step of fourstage process where cardiac transplantation is the ultimate goal. Considering cardiac transplantation as an inevitable occurrence is most probably an expectation of many cardiologists. However, this conviction does not appear to be reflected in how we counsel our patients, at least at the initial stages of management when the details of Fontan stages are discussed. Such discussions include little or no reference to the ultimate and likely need for cardiac transplantation in a large percentage of these patients.

Newburger et al. in studying the outcome of various shunts during the Norwood procedure demonstrated an overall mortality of $36 \%$ [1]. Siffel et al. documented a long-term 
mortality rate of $48 \%$ in HLH [2]. Other studies showed a more optimistic outcome [3]. In a more recent article, Rogers et al. studied the mortality rate in children born with HLH syndrome in England and Wales during the time period of 2000-2015. They identified that the 1- and 5-year survival in the early era was 56.6 and $53.8 \%$, respectively. On the other hand, during the recent era (2006-2015) when the hybrid procedure was available the 1- and 5-year survival rates were 63.9 and $58.1 \%$ for the traditional patients versus 47.2 and $47.2 \%$ for the hybrid patients [4]. In a study of patients surviving the Fontan procedure in Australia and New Zealand obtained from a binational population-based registry including all pediatric and adult cardiac centers, 1006 survivors of the 1089 patients who underwent the Fontan procedure were investigated. Patients with HLH syndrome formed a small portion of this population. The investigators found that HLH was the primary predictor of Fontan failure and the 10 -year freedom from failure was $79 \%$ for HLH syndrome versus $92 \%$ for other morphologies [5]. Therefore, if one is to take in consideration the added interstage mortality of the Fontan procedure one can estimate that at 10 years after the initial step of the Fontan procedure, the percentage of survival is likely to be $<50 \%$, consistent with other studies. Furthermore, as those patients grow older in their twenties and beyond, one should expect an ever decreasing rate of transplant-free survival.

If one is to accept the premise that cardiac transplantation is a likely event in HLH patients after years of a Fontan circulation, is it reasonable then to think of it as a fourth stage, just as we think of the other stages of Fontan. A Norwood stage I or Glen shunt procedures are rarely thought of as an ultimate goal. Instead they are necessary steps towards the completion of the systemic venous to pulmonary arterial connection, or stage three of the Fontan procedure. Is it then conceivable that we can develop criteria which would trigger the initiation of cardiac transplantation consideration as the fourth stage in managing their congenital heart surgery? Such a strategy would allow for timely intervention prior to development of multi-organ failure known to occur in Fontan patients. Naturally I am not advocating cardiac transplantation where it is not needed, the shortage of organs for transplantation is a significant obstacle as it is, let alone one has to maintain the "Do no harm" approach to any medical management and finally life after transplantation has its own limitations and complications. However, I am advocating that we come to realize that cardiac transplantation, or any yet to be discovered treatment modality, is inevitable for HLH patients at an increasing rate with advancing age, and as such it has to be thought about and prepared for when indicated. Perhaps it is not a stretch to think of the Fontan procedure, just as we do with the Norwood stage I and Glen shunt procedure as a stage which has an "expiration date."
Another area where a shift in focus is much needed is current practice of post-operative care. Much of my practice has been in intensive care units, but even this did not allow me to appreciate the physical and psychological impact we, by necessity, impose on our patients. Things as minor as a blood draw or an IV placement is traumatizing and to a certain extent health care providers in general and perhaps consultants in particular may not appreciate or give it much thought. Much of our goals are focused on discharging patients with minimal morbidity and mortality and not the psychological well-being of these children while in the intensive care unit. Reliable non-invasive assessment tools, similar to pulse oximetry, are few as we continue to rely on blood draws for accurate data. The same is true for pharmacotherapy where intravenous delivery is still the reliable mode in most instances, at least in the early post-operative care. Reliable oral and transdermal medication delivery seems to progress at a snail pace with limited investment in non-invasive medication delivery.

We have made significant strides in helping children and adults with HLH syndrome. More can be done as we listen to our patients, advocate for research to enhance post-operative care with non-invasive monitoring and medication delivery systems as part of improvement strategies which take into account outcomes as well as patients' psychological well-being.

Ra-id Abdulla, MD

Editor-in-Chief

\section{References}

1. Newburger JW, Sleeper LA, Frommelt PC, Pearson GD, Mahle WT, Chen S et al (2014) Transplantation-free survival and interventions at 3 years in the single ventricle reconstruction trial. Circulation 129:2013-2020

2. Siffel C, Riehle-Colarusso T, Oster ME, Correa A (2015) Survival of children with hypoplastic left heart syndrome. Pediatrics. https ://doi.org/10.1542/peds.2014-1427

3. Tweddell JS, Sleeper LA, Ohye RG, Williams IA, Mahony L, Pizarro $C$ et al (2012) Intermediate-term mortality and cardiac transplantation in infants with single-ventricle lesions: risk factors and their interaction with shunt type. J Thorac Cardiovasc Surg 144:152-159

4. Rogers L, Pagel C, Sullivan ID et al (2018) Interventional treatments and risk factors in patients born with hypoplastic left heart syndrome in England and Wales from 2000 to 2015 . Heart 0:1-8. https://doi.org/10.1136/heartjnl-2017-312448

5. d'Udekem Y, Iyengar AJ, Galati JC et al (2014) Redefining expectations of long-term survival after the Fontan procedure: twentyfive years of follow-up from the entire population of Australia and New Zealand. Circulation 130(11 Suppl 1):S32-S38. https://doi. org/10.1161/CIRCULATIONAHA.113.007764 